\title{
BMJ Open KEPT-app trial: a pragmatic, single- blind, parallel, cluster-randomised effectiveness study of pelvic floor muscle training among incontinent pregnant women: study protocol
}

\author{
Sherina Mohd Sidik (D) , ${ }^{1}$ Aida Jaffar (1) , ${ }^{1,2}$ Chai Nien Foo (D) , ${ }^{3}$ \\ Noor Azimah Muhammad, ${ }^{4}$ Rosliza Abdul Manaf, ${ }^{5}$ Siti Irma Fadhilah Ismail, ${ }^{1}$ \\ Parwathi Alagirisamy, ${ }^{6}$ Amalina Farhi Ahmad Fazlah, ${ }^{7}$ Zailiza Suli, ${ }^{8}$ \\ Felicity Goodyear-Smith (1) ${ }^{9}$
}

To cite: Sidik SM, Jaffar A, Foo CN, et al. KEPT-app trial: a pragmatic, single-blind, parallel, cluster-randomised effectiveness study of pelvic floor muscle training among incontinent pregnant women: study protocol. BMJ Open 2021;11:e039076. doi:10.1136/ bmjopen-2020-039076

- Prepublication history and additional material for this paper is available online. To view these files, please visit the journal online (http://dx.doi.org/10. 1136/bmjopen-2020-039076)

Received 06 April 2020 Revised 17 November 2020 Accepted 13 December 2020

Check for updates

(c) Author(s) (or their employer(s)) 2021. Re-use permitted under CC BY-NC. No commercial re-use. See rights and permissions. Published by BMJ.

For numbered affiliations see end of article.

Correspondence to

Professor Sherina Mohd Sidik; sherina@upm.edu.my

\section{ABSTRACT}

Introduction Pelvic floor muscle training (PFMT) strongly recommended to incontinent pregnant women. The Kegel Exercise Pregnancy Training-app trial is a multicentre cluster-randomised study aims to assess the effectiveness and its cost-effectiveness of the mobile app guidance in PFMT among incontinent pregnant women.

Methods and analysis 370 pregnant women (aged 18 years old and above) will be recruited with International Consultation on Incontinence Questionnaire-Urinary Incontinence-Short Form. Ten clusters (primary care clinics) will be randomly assigned to either PFMT or usual care in a 1:1 ratio by an independent researcher (sealed envelope). The primary outcome will be urinary incontinence, and the secondary outcomes (quality of life; PFMT adherence, psychological status and mobile apps' usability) will be assessed at four measurement time points (t0: baseline) and postintervention (t1: 4 weeks, t2: 8 weeks and t3: 8 weeks postnatal). T-test analysis will determine any significant differences at the baseline between the control and intervention groups. The mixed-model analysis will determine the effectiveness of the intervention at the population-average level for both the primary and secondary outcomes. For the costeffectiveness analysis, expenditures during the study and 6 months after the intervention will be compared between the groups using the multiway sensitivity analysis. The recruitment planned will be in December 2020, and the planned end of the study will be in August 2021.

Ethics and dissemination This study protocol was approved by the Ethics Committee for Research Involving Human Subjects, Universiti Putra Malaysia (JKEUPM-2019-368) and Medical Research and Ethics Committee (MREC), Ministry of Health Malaysia, NMRR19-412-47116 (IIR) with the ANZCTR registration. This study will obtain informed written consent from all the study participants. The results which conform with the Consolidated Standards of Reporting Trials and the Recommendations for Interventional Trials will be published for dissemination in peer-reviewed journals and conference proceedings.

\section{Strengths and limitations of this study}

- To our knowledge, this study is the first pragmatic study at the primary care level to examine the effectiveness of pelvic floor muscle training (PFMT) delivered via a mHealth app among incontinent pregnant women (primigravida and multigravida).

- The mHealth app was designed based on the Cognitive, Opportunity, Motivation-Behaviour embedded in Behavioural Change Wheel using the Intervention Mapping framework.

- The study will include only 10 clusters in which all will be in one district in the state of Selangor, Malaysia. Therefore, the decision of whether the findings can be generalised to another district remains to be further studied.

- The PFMT adherence among pregnant women as a secondary outcome will only be measured until 8 weeks postnatal.

- The self-efficacy assessment will be done via a validated questionnaire without any objective assessment, which may not provide accurate results.

Trial registration number ACTRN12619000379112.

\section{INTRODUCTION}

Urinary incontinence (UI) occurs when a person is unable to control urine, leading to its involuntary leakage. ${ }^{1}$ It is stated as one of the greatest burdens in 2018 by affecting about 423 million globally. ${ }^{2}$

Pregnancy consists of three trimesters: first (early until week 12 of pregnancy); second (week 13 to week 27 of pregnancy) and third trimester (28 weeks onwards). ${ }^{3}$ UI significantly increases in the second and then the third trimesters, as well with an increased number of pregnancies. ${ }^{4}$ A study in Norway 
composed of 43279 pregnant women reported that parity was a strong risk factor for UI with the (OR: 2.1, 95\% CI 2.0 to 2.2) with the increased prevalence of UI from $35 \%$ before pregnancy to $67 \%$ during week $30 .{ }^{5}$ Similarly, one primary care clinic reported prevalence of UI was $40.1 \%$ $(\mathrm{N}=440)$ with double the risk of UI among multigravida (OR 2.01, 95\% CI 1.24 to 3.26) ${ }^{6}$

There are three types of UI: stress UI (SUI) with increased abdominal pressure leading to extra stress on the bladder; urge UI (UUI) with an unstable or overactive bladder and mixed UI (MUI) (a combination of stress and urge $).{ }^{78} \mathrm{SUI}$ is the most common during the pregnancy due to excessive stress on the bladder from the uterus enlargement, with $17 \%-54 \%$ of the female population experiencing their first UI during pregnancy. ${ }^{9} 10$ A study among primigravida reported that SUI in the third trimester was the most prevalent $(64.8 \%)$, followed by MUI (24.8\%) and UUI (6.7\%). ${ }^{11}$ A similar finding reported among mixed parity at a primary care clinic with a majority of them having SUI $(75.6 \%, \mathrm{n}=136){ }^{6}$

Studies have shown that pelvic floor muscle training (PFMT) or Kegel exercise is most effective in treating SUI and MUI, by strengthening the pelvic floor muscles. ${ }^{8}{ }^{12-15}$ PFMT has been recommended as the first-line treatment in UI. ${ }^{8}$ There were a few barriers for pregnant women to do PFMT, for example; poor knowledge, attitude and practice towards PFMT $^{6} 16$ and to remember the daily PFMT.$^{17}$ mHealth app has shown its importance (Level of evidence II) as it enables reminders and improvement to the accessibilities of PFMT for the patients. ${ }^{18}$

Several studies reported promising results using mHealth apps in managing UI. ${ }^{19}$ Similarly, a recent randomised controlled trial (RCT) on the effectiveness of a mHealth app-based audio guidance home training PFMT among primipara (after receiving personal PFMT by a physiotherapist) showed a positive outcome. The study reported with significant improvement in SUI, especially after 3 -month training with $\beta=-5.520(p<0.001)$ from 54 primiparas. ${ }^{20}$

In conclusion, PFMT via mHealth app shows improvement in UI due to PFMT adherence. The essence in managing UI is to be able to adhere to the correct PFMT. $^{21}$ Furthermore, mHealth app improves the accessibility of PFMT to pregnant women as supervised PFMT is time-consuming and involves healthcare workers which may lead to less cost-effectiveness and poor adherence to training.

Despite the positive outcomes of PFMT as the treatment for incontinent pregnant women, a recent Cochrane review reported an uncertain outcome effect. ${ }^{22}$ The risk of bias and low-quality evidence skewed the pooled results into the uncertain effect. ${ }^{22}$ This review may steer physicians, stakeholders and policy-makers away from promoting PFMT as the treatment for UI among pregnant women.

Therefore, it is vital to conduct a good quality pragmatic, single-blind cluster RCT (CRCT) to determine the effectiveness of PFMT in primary care (usual care) settings. The findings from this study bridge the implementation gaps as it balances between internal validity and generalisability. ${ }^{23}$ Hence, it enables us to provide essential evidence about PFMT's effectiveness as the treatment of choice for pregnant women with UI in the healthcare system.

\section{Pragmatic effectiveness trial of PFMT as a treatment for} pregnant women: the Kegel Exercise Pregnancy Training mHealth-app trial

The Kegel Exercise Pregnancy Training mHealth app trials (KEPT-app) study is a multicentre cluster-randomised trial. The aim is to assess the PFMT delivered via the mHealth app as a treatment approach among pregnant women in community-based healthcare in comparison to standard-care (usual care) antenatal clinic follow-up. We examine the following hypotheses: at 1-month (early third trimester), 2-month (late third trimester) and 8 weeks postnatal (early postnatal) follow-up, the intervention groups (IGs) show significant improvement in their UI symptoms, quality of life (QoL), knowledge, attitude and practices of PFMT, adherence to PFMT and self-efficacy in PFMT compared with the control groups (CGs) which receive the usual care (waitlist groups).

\section{Aim}

This study aims to determine the effectiveness of mHealth app guidance PFMT on the treatment of UI in pregnant women. The app is named as the KEPT-App. The primary objective is to determine whether KEPT-app results in improving UI in pregnant women. The secondary outcomes are QoL, knowledge, attitude and practices of PFMT, PFMT adherence, self-efficacy towards PFMT, psychological status and usability of the KEPT-app.

\section{METHODS AND ANALYSIS \\ Study design}

This study is a pragmatic, single-blind, cluster CRCT involving 10 primary care clinics in Hulu Langat district, which is one of the nine districts in the state of Selangor, Malaysia. The district chosen for this study has the largest number of clinics with the second-highest number of deliveries in Selangor. Selangor had the highest number of antenatal cases in 2017 with more than 1 million pregnant women attendees. ${ }^{24}$

This cluster trial is based on the administrative unit (Hulu Langat District Health Office) which enables to clearly map the boundaries between the neighbouring district, decrease concerns over contamination and reduction between-cluster variability. ${ }^{25}$

Each primary care clinic will be randomised to either the intervention or CG for at least 16 weeks as the intervention starts at 28 weeks until delivery at 36 weeks and PFMT continues up to 8 weeks postnatal) as illustrated in figure 1 .

This study will use cluster randomisation with allocation concealment in order to reduce selection bias. ${ }^{26}$ The 


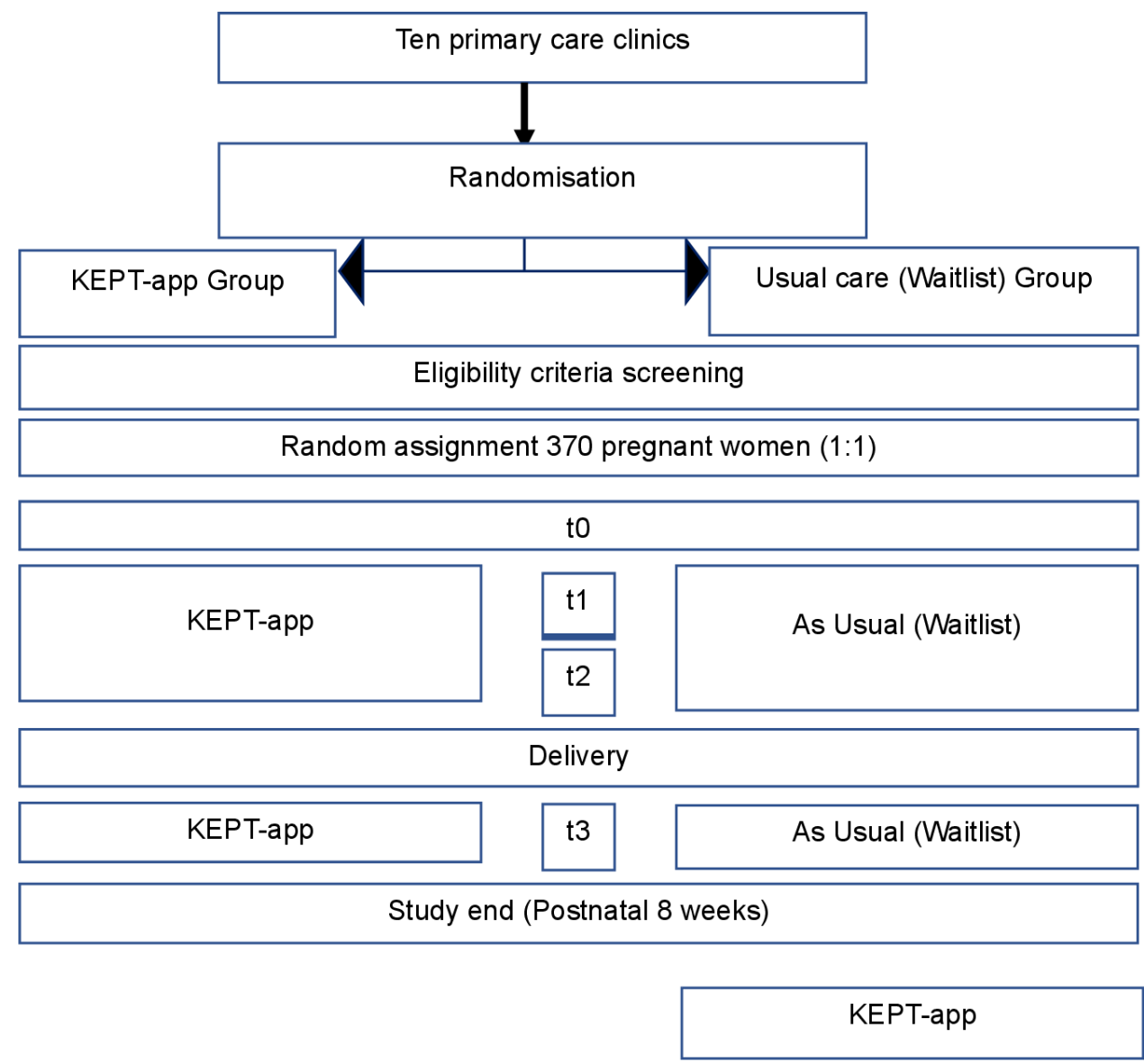

Figure 1 Study design. KEPT-aPP, Kegel Exercise Pregnancy Training mHealth app.

name of 10 clinics in Hulu Langat will be written in separate envelopes and concealed from the researchers. A blinded independent researcher will randomly assign ten clusters (primary care clinics) to one of two groups: IGs which receives KEPT-app and CGs which receive the treatment as usual (waitlist group). He/she will pick one by one, envelopes with the five clinics name tagged as either IGs or CGs each in alternate sequence. Therefore, there will be five clinics in each group which as per recommendation to have at least four clusters per arm. ${ }^{25} 27$

The total participants of 370 will be randomly allocated in a 1:1 ratio to either the IGs or the CGs according to their usual antenatal care clinic follow-up. The number of women recruited from respective clinics will depend on the total number of antenatal patients in each clinic: a higher proportion of participants will be recruited from clinics with a larger number of antenatal patients and vice versa.

The interventions will start immediately after the baseline data ( $\mathrm{t} 0$ : baseline) has been collected. The participants will download the KEPT-app instruction for a short introduction briefing and are encouraged to contact the research assistants (RAs) for further clarification if needed. The PFMT video and the training will start on the same day the KEPT-app is downloaded, and the diary with the reminder will start the next day.
After 4 weeks, both groups will receive follow-up assessments as t1. Two more follow-up assessments will be delivered at t2: 8 weeks (after treatment initiation) and t3: 8 weeks postnatal. Two weeks before the date of the assessment, a booster (cue to watch the video) will be delivered to the IGs. All participants in both groups will receive three follow-up assessments (t1: 4 weeks after treatment initiation/enrolled into the CGs, t2: 8 weeks after treatment initiation/enrolled into the CGs, t3:8 weeks postnatal, respectively), primary and secondary outcome measures will be assessed via online assessment as in table 1 and figure 2.

KEPT-app trial will be a single-blind study whereby the researcher and assessor will be blinded to the group affiliation. The participants will not be blinded as they are aware of which groups they belong to.

This study protocol is designed and reported according to the recommendations in the Standard Protocol Items: Recommendations for Interventional Trials (SPIRIT) ${ }^{28}$ and Consolidated Standards of Reporting Trials (CONSORT) statements extensions for pragmatic trials, ${ }^{29}$ CONSORT CRCTs ${ }^{27}$ (see online supplemental file 1), and checklist from the Pragmatic-Explanatory Continuum Indicator Summary-2. ${ }^{30}$

This pragmatic design will give a real-world view and provide information on the internal validity of the 
Table 1 Overview outcome measures

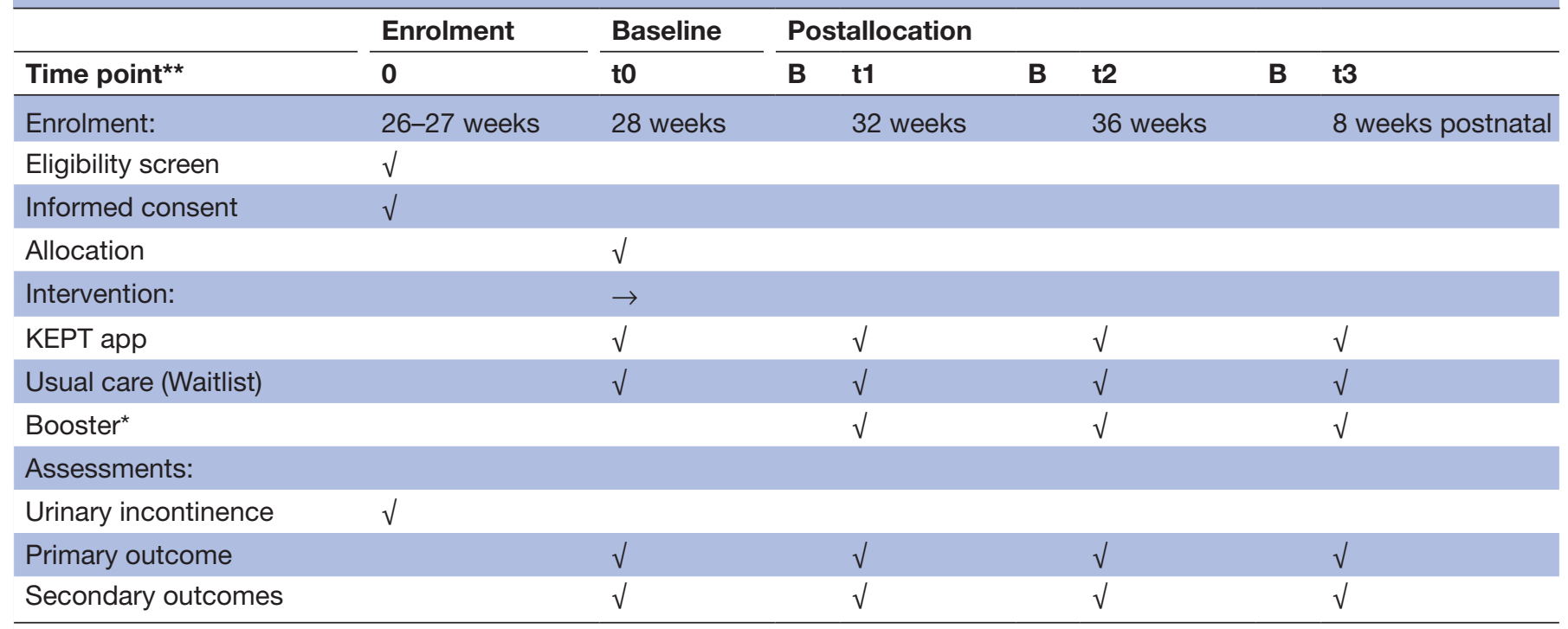

B: Booster (Cue to revisit the video)

KEPT, Kegel Exercise Pregnancy Training mHealth.

intervention in improving the intended problem. ${ }^{31}$ In this context, the effectiveness of the KEPT-app in improving the UI symptoms experience of pregnant women. There are four steps to ensure the pragmatism of a study with nine domain indicators. ${ }^{30}$ This present study scores 4.6, which is high pragmatic (see online supplemental file 2 ). The journal abstract for this study is according to the recommendation from the CONSORT extension for journal abstracts ${ }^{32}$ (see online supplemental file 3 ).

\section{Participants eligibility}

We will recruit a total of 370 pregnant women presenting to 10 primary care clinics in Hulu Langat district, Selangor state in Malaysia. Each clinic will be expected to recruit between 20 and 60 pregnant women, proportionate with their pregnant women attendances based on the sample size calculation.

Participants who are more than 18 years old, primipara and multiparas at 26-27 weeks' gestation with UI scores of 3-18 according to the International Consultation on Incontinence Questionnaire-UI-Short Form are eligible to participate in the study. They are Malaysian citizens who consist of Malay, Chinese, Indian and native ethnicities, and will be included in this study, as the main language used in this study will be Malay, which is the national language of Malaysia.

Pregnant women with a chronic medical problem(s) before pregnancy (diabetes, hypertension, HIV positive, a neurological condition affecting bladder control, stroke, pelvic organ prolapse) will be excluded as UI in this condition should not be treated by PFMT only. In addition to the exclusion list are complicated pregnancies or conditions which are not advisable to practise PFMT (such as pre-eclampsia, persistent bleeding, preterm, labour, incompetent cervix, acute febrile infection, fetal growth restriction or placenta previa and cephalopelvic disproportion).

The researchers will be blinded to the participants' responses and their group allocation. RAs will be responsible for the recruitment of the participants and assist them with the electronic informed consent and data collection. The RAs will have a short training on communication skills, the KEPT-App and short message system (SMS) link for questionnaires and will contact with the main researcher to discuss any issues during the recruitment or data collection.

The RAs will screen for participants at 26 weeks or 27 weeks of pregnancy based on the information written in their antenatal books. Those who fulfil the study criteria will be invited using consecutive sampling method by recruiting every patient who meets the selection criteria within the recruitment period of 3 months. ${ }^{33}$ We acknowledge the commitment and the importance of participant retention and their adherence participating in our study. We will give the CG the KEPT-app once they complete the study after 8 weeks postnatal. The detail of the recruitment, as illustrated in figure 3 .

The RAs will explain to the participants they can leave this study at any time when they demand to do so, without any consequences. If any pregnant women have any health issues during the research, they will be encouraged to seek help from their healthcare professionals during antenatal follow-up or at any primary care clinic to receive any treatment. Evidence has shown no harm to perform PFMT among pregnant women. ${ }^{34}$

\section{Intervention}

The KEPT-App will guide correct techniques of PFMT and encourage pregnant women to adhere to the PFMT. The intervention consists of a KEPT-app implementation 


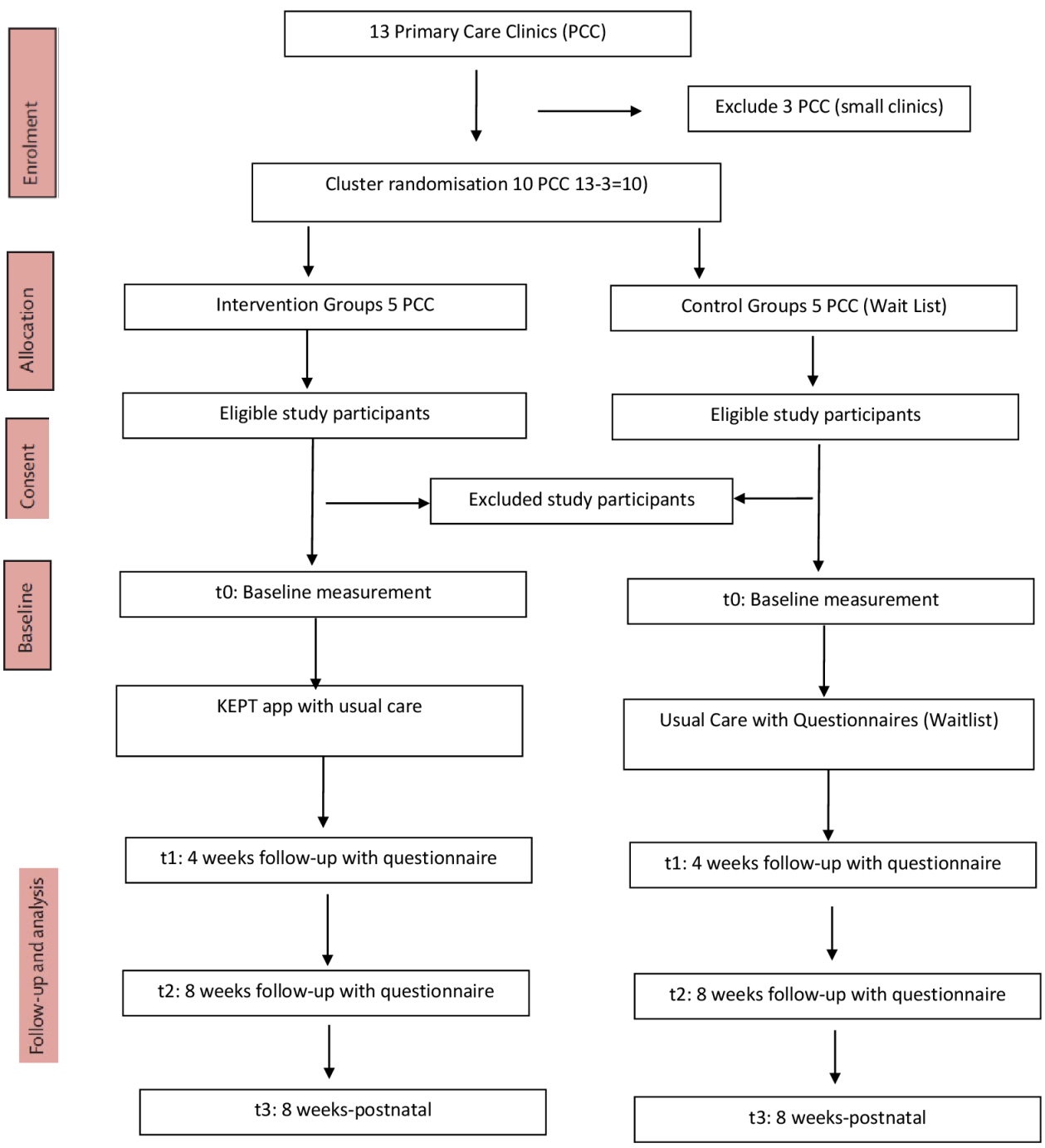

Figure 2 CONSORT 2010 flow diagram. CONSORT, Consolidated Standards of Reporting Trials.

which will be started at 28 weeks of gestation, and its effectiveness will be evaluated at 8 weeks postnatal.

The IG will receive a KEPT-app additional to the usual care while the CG will receive usual antenatal care and SMS with the link to the questionnaires. During recruitment and before obtaining the consent, RAs will explain the aims, justification, and process of the intervention to the participants.

The KEPT-app will be developed according to an 'Intervention Mapping'. It is a guided and systematic approach protocol which incorporates the theory-based and evidence-based health promotion programmes in developing the health promotion intervention. Intervention Mapping has six steps which are ${ }^{1}$ the needs assessment (logic model), ${ }^{2}$ the definition of the programme outcome and change, ${ }^{3}$ the choice of the most appropriate theorybased intervention methods to design the programme, ${ }^{4}$ the programme production, ${ }^{5}$ implementation of the programme, and $^{6}$ evaluation of the programme. ${ }^{35} 36$

KEPT-app is a design from the Cognitive, Opportunity, Motivation-Behaviour (COM-B) theory embedded in the Behaviour Change Wheel (BCW) Framework to adopt
PFMT as the new habit. The BCW Framework are recent developments that provide a systematic and theoretical basis for understanding and changing behaviour. ${ }^{37}$ It has eight steps to bring theory-based tool together into a coherent intervention design: define the problem in behavioural terms, select the target behaviour, specify the target behaviour, identify what needs to change, identify the intervention functions, identify the policy categories, identify the behaviour change techniques and identify the mode of delivery. ${ }^{37} 38$

In the (COM-B) theory, KEPT-app will use the social cognitive theory as one of its behavioural change theory, which states that behavioural change is dependent on a person's autonomy and self-efficacy is essential for PFMT adherence. ${ }^{39}$ A pregnant woman is more likely to adhere with PFMT if she has good self-efficacy about the exercise. PFMT techniques will be delivered using an instructional video which demonstrates different positions, guides the users on the correct method, and explains the common mistakes and myths of PFMT. A certified, experienced physiotherapist conducts the demonstration in line with the recommendation guidelines. ${ }^{21} 40$ The KEPT-app 
Posters and Pamphlet announcement on study

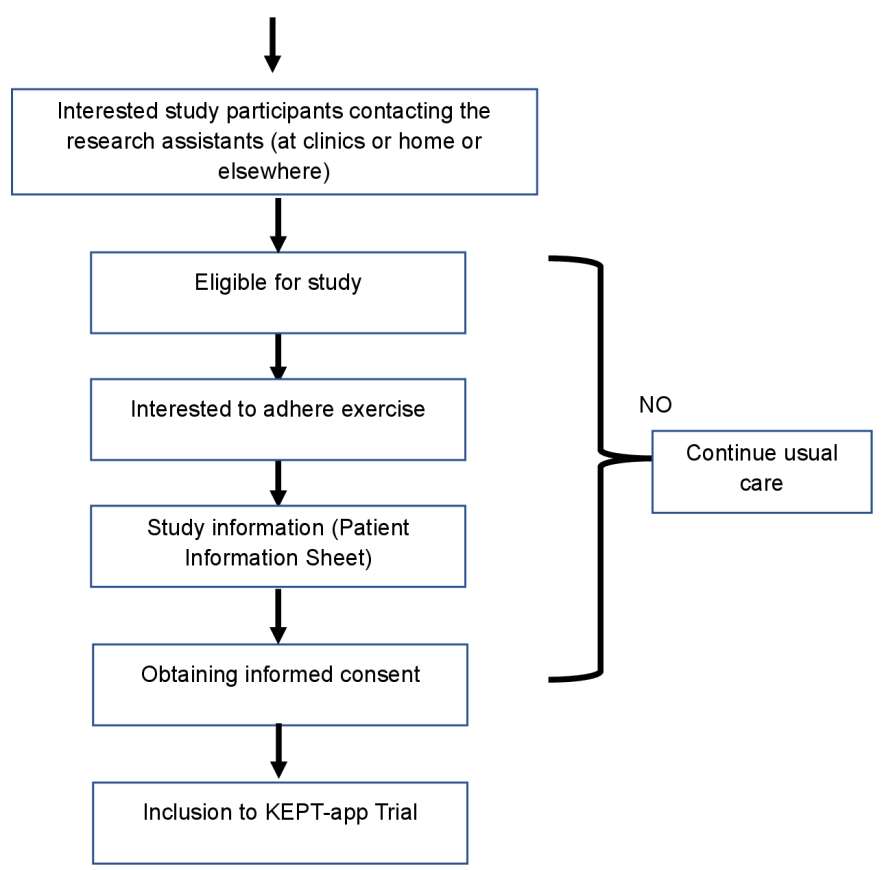

Figure 3 Recruitment flow chart.

development in the process of manuscript writing to publish in the peer-reviewed journal, as recommended in the Good Practice Guideline Health Apps and Smart Devices (Mobile Health or mHealth). ${ }^{41}$

After participants have understood the video, they will enter the training mode of the KEPT-app. This training mode will guide them step by step with the instructor's voice to be ready at the position, take a deep breath and contract for a few seconds (to get the feel of the contraction). After they have understood the correct feeling of their muscles contracting, they will move to the next level, which is the training mode of $10 \mathrm{~s}$. The aim is to be able to do PFMT for $10 \mathrm{~s}$ each time (a few seconds resting) for ten times per cycle with a total of three cycles per day.

The study participants will move out of the training mode, once they can perform the PFMT confidently. They proceed to the adherence phase, where the aim is to perform ten cycles three times a day. KEPT-app will record the exercise activities during the study period to capture the actual adherence rate with a reminder occurring on the screen if no activities are recorded.

Participants will be able to track their improvement in the UI symptoms and PFMT adherence. KEPT-app will undergo its validity and usability testing before its implementation in this study according to the recommendation by the CONSORT extension PRO. ${ }^{42}$

\section{Sample size calculation}

The sample size for this interventional phase is using the individually randomised trial formula of continuous outcome $\mathrm{N}=2\left(Z_{\alpha / 2}+Z_{\beta}\right)^{2}\left(\sigma^{2}\right) /\left(\mu_{1}-\mu_{2}\right)^{2}$. $^{43}$

The details of the formula whereby $Z_{\alpha / 2}$ and $Z_{\beta}$ are the standard normal values corresponding to the upper tail probabilities of $\alpha / 2$ and $\beta$ respectively. The $\alpha$ is the twosided significance level and $1-\beta$ is the power, $\mu 1$ and $\mu 2$ are the means in the two arms, and $\sigma$ is the SD of the outcome.

Clustering method involves multiplying of the sample size that requires to be as equally as an individually randomised trial, multiplying with the design effect or formerly known as variance inflation factor. ${ }^{44}$ The formula for the design effect is: Deff $=1+\rho(m-1)$, where $\rho$ is the intercorrelation coefficient (ICG), and $m$ is the cluster size. $^{45}$

The ICC is a between-cluster variation measurement or the homogeneity of individual measures within a cluster. The ICC's level is according to the setting and type of outcome. ${ }^{46}$ For process variables, the ICCs are higher than those for patient outcomes, and similarly, the ICCs of the secondary care are higher than those from primary care. ICCs for process variables in primary care are of the order of 0.05-0.15 with the ICCs for patient outcomes in primary care are generally lower than $0.05 .^{46}$

However,if the actual cluster sizes are unknownn, the design effect needs to be readjusted according to the coefficient of variation of the cluster sizes, $C V$, the formula: $c v_{c}=s_{c} / \dot{m}$ where $s_{c}$ is the $\mathrm{SD}$ of cluster sizes and $\dot{m}$ is the average cluster size.

The $c v$ is unknown; hence we can estimate using the likely minimum and maximum cluster size. ${ }^{45}$ The $s_{c}$ is estimated by $\mathrm{SD}=$ CSrange/4. The $\mathrm{SD}$ in this study is $40 / 4=5$ (CS range of 40 is the estimated difference between the cluster size according to the previous attendees to the clinics in Hulu Langat). The formula to get the is $c v=\mathrm{SD} / \dot{m}^{47}$ where the mean cluster size from previous CRCT at our primary care clinics was $63 .^{48}$ Following this, the design effect was then calculated by the formula Deff $=1+\left[\left(c v_{c}^{2}+1\right) \dot{m}-1\right] \rho .{ }^{474}$ Therefore, the design effect (Deff) in this study is $1+[(1.03) 63-1]=4.2$.

In summary, referring to table 2 , the sample size for this study is 368 and after round-up is 370 pregnant women.

\section{Measures}

This summary of the primary and secondary outcome assessment is given in table 3. Participants will be given the online assessments at four measurement time points (t0-t3) either via KEPT-app (IGs) or google-form link via SMS (CGs). At t3, there will be an additional assessment on the usability of the KEPT-app only for the IGs participants.

\section{Cost efficiency}

The expenditures to purchase incontinence aids will be collected to assess the cost of the UI in both groups during the study. As this will be from the participants' perspective, an estimate of the cost for their time will be included. Therefore, the participants will check back their PFMT record on time spent during the study. As for the usual care group, the assumption will be that the time spent remains constant during the study. This study will use its gross hourly wages to estimate the time cost. This study 


\begin{tabular}{|c|c|c|c|c|c|c|c|}
\hline No & Variables & $\sigma$ & $\rho$ & $\mu 1-\mu 2$ & Deff & Non-respondent (30\%) & Attrition rate $(20 \%)$ \\
\hline 1 & $\begin{array}{l}\text { UI Severity } \\
\text { ICIQ-UI SF }\end{array}$ & 2.56 & 0.05 & 2.52 & 4.2 & 88 & 212 \\
\hline 2 & $\begin{array}{l}\text { Quality of Life } \\
\text { ICIQLuts Qol }^{64}\end{array}$ & 4.95 & 0.05 & 3.71 & 4.2 & 153 & 368 \\
\hline 3 & Knowledge PFMT ${ }^{65}$ & $3.4^{*}$ & 0.05 & 4.67 & 4.2 & 45 & 108 \\
\hline 4 & Attitude PFMT ${ }^{65}$ & $3.7^{*}$ & 0.05 & 3.77 & 4.2 & 82 & 198 \\
\hline 5 & Practices PFMT ${ }^{65}$ & $1.73^{*}$ & 0.05 & 3.45 & 4.2 & 22 & 52 \\
\hline
\end{tabular}

${ }^{*} \mathrm{SD}$ using Lemeshow et a/ ${ }^{66}$ calculation.

ICIQ-UI SF, International Consultation on Incontinence Questionnaire-Urinary Incontinence-Short Form; PFMT, pelvic floor muscle training; QoL, quality of life.

will use the mixed-model analysis to compare treatment effects between groups using the available data on the UI and QoL. Sensitivity analysis will be performed according to the data available.

\section{Data collection}

Quantitative data

This study adheres with the Consolidated Standards of Reporting Trials flow chart to recruit the participants, assess them and report findings.

\section{Data for primary and secondary outcomes}

All data will be collected digitally via mobile apps and google documents (with a reminder a week before the data collection's date) and will be kept in data storage. Once the participants register into the system, they will be assigned a unique identification number.

\section{Validation}

The questionnaires used in this study have been translated and validated locally. The KEPT-app will be validated in its usability aspect among forty pregnant women who are not involved in the intervention study. The educational video will be validated using Patient Education Materials Assessment Tool audiovisual tool. The results of this validation study will be published either in conference proceedings or in a peer-reviewed manuscript.

\section{Planned statistical analyses}

This study will report the data at baseline ( $\mathrm{t} 0$ ), post- 1 month (t1), post-2 months (t2) and 8 weeks postnatal (t3) after the intervention. The data will be compared at the baseline to determine any significant differences between the control and IGs using the t-test. This study will apply the

\section{Table 3 Study outcomes KEPT-app trial}

Primary outcome Information

1. International Consultation on Incontinence Questionnaire-Urinary Incontinence Short Form (ICIQ-UI SF ${ }^{67}$ )

To measure the reduction in urinary incontinence score at 1 month, 2 months and postnatal in intervention and control groups. Therapy success is defined as absence of UI or change from baseline of at least three points on the ICIQUI-SF at 8 weeks postnatal.

\section{Secondary outcomes}

1 Knowledge, Attitude and Practice towards PFMT ${ }^{16}$

2. Exercise Adherence Rating Scale (EARS). ${ }^{68}$

3. Self-Efficacy Scale For Practicing Pelvic Floor Exercise Questionnaire (SESPPFE)

4. ICIQ Urinary Incontinence-Lower Urinary Tract Symptom quality of life (ICIQ-LUTSqol) ${ }^{67}$

5 The Patient Health Questionnaire-9 (PHQ-9) consists of nine self-reported questionnaires, based on DSM-IV criteria for major depression. ${ }^{69-71}$

6 The Generalised Anxiety Disorder scale (GAD-7) has been developed The improvement of GAD-7 scores. to be used in primary care to diagnose the GAD, panic disorder, social anxiety disorder and post-traumatic stress disorder ${ }^{72-75}$

KEPT app, Kegel Exercise Pregnancy Training mHealth app; PFMT, pelvic floor muscle training. 
intention-to-treat principle appropriately for primary and secondary outcomes.

The statistical analyses will be as recommended by SPIRIT 2013, which is the intention-to-treat basis. ${ }^{50}$ The participants who have completed the baseline $(\mathrm{t} 0)$ will be included in the analyses, and their final observations will be carried forward. For example, if the pregnant woman at Clinic A call and request for drop out, the researchers will ask for her main symptoms and outcomes at that time. This study is a single-blind study whereby the researchers and assessor (who performs the statistical analysis) are blinded. Therefore, the IGs and CGs will only be revealed after the analysis of the primary outcome has completed.

Since this study uses multi-centres (primary care clinics), the random effect may exist. Therefore, generalised estimating equation (GEE) analysis will determine the effectiveness of this intervention programme at the population average as proposed by SPIRIT 2013 guideline. ${ }^{28}$ GEE will be able to handle missing data with multiple imputations. ${ }^{51}$ The IBM SPSS Statistics for Windows, V.25 software is used for all statistical analyses. All statistical tests are two sided with $\mathrm{p}<0.05$ considered as statistically significant.

\section{Methodological issues \\ Cluster RCT}

The KEPT-app trial will recruit patients at the population level with intervention delivered individually; hence it is a combination of the population and individual intervention. The cluster size for each arm is five clusters and has achieved its minimum recommended by CONSORT 2010 statement. ${ }^{27}$ Unfortunately, this study has two arms with a total of 10 clusters, and this could be at risk of type I error. ${ }^{52}$ Therefore, the authors suggest using GEE with a small-sample correction to accommodate this error. ${ }^{53} 54$

This study aims to improve the antenatal care system at primary care level, and cluster-randomised trial approach is preferred despite the KEPT-app being delivered individually. By evidence of treatment success among nonpregnant women with UI using a mobile app (Tät), there is an advantage to deliver this intervention at the population level. ${ }^{55}$

\section{Pragmatic approach}

Pragmatic RCT approach is to ensure the effectiveness study on KEPT-app as closely as possible to the real antenatal clinic in primary care practices. Unfortunately, this study only includes one district which consists of ten primary care clinics which put the external validity at risk. The small number of clusters which is due to limited resources will affect the internal validity of this study. However, it is essential to find a balance between internal and external validity in a pragmatic study while strictly adhere to its methodological requirements and recommendations. ${ }^{2856-58}$

\section{Patient and public involvement}

Participants and members of the public will be involved in the study design and the conduct of the trial. The feedback and expectations from the participants of the KEPT-app in terms of designs and content will be analysed. The researchers will conduct pilot testing to improve its version before trial implementation to enhance its cost-effectiveness.

\section{Data management and clinical trial unit support}

The data will be collected via the KEPT-app link and SMSlink and entered directly into the trial database. Data gathered from the KEPT-app enables the participants to access their data, monitor their UI symptoms and act as PFMT motivation. The data will be stored in the cloudbased Azure storage.

The research progress will be shared to the Universiti Putra Malaysia and ethical committee 6 monthly. However, the researcher will keep the research notes for 5 years following the publication of the study's results to provide background information for future mHealth applications. Technical appendix, statistical code, and dataset will be available from the Dryad repository, DOI 10.13140/RG.2.2.34968.34568.

\section{Data privacy, protection, security and access}

A unique study identification data (ID) code will be used to ensure anonymity and confidentiality. This unique ID will be used to identify participants and the group they belong to. The participants' usernames, patient's registration number and other personal information, will never be linked to their responses nor personal data. The researcher is responsible for ensuring data safety. The participants will have access to the results of their assessments. They will be able to monitor their UI progression via their apps.

To protect the confidentiality, researcher assistants (RA) will discuss confidentiality issues with all participants. In addition to this, all codes, notes, or files, will be encrypted or encoded and stored in only one computer. The RA will ensure that all research files, notes and codes are password-protected, and these files and passwords will be only accessible to one designated RA. The researcher will ensure the maintenance of confidentiality during the study with the compliance by the Data Privacy Act to be highlighted to all study participants. ${ }^{59}$

\section{Mobile apps data protection and security}

The data exchange between client and server will be encrypted using the Rivest-Shamir-Adleman algorithm. ${ }^{60}$ In addition to encrypting the data transmitted between client and server, Hypertext Transfer Protocol Secure is enabled and powered by Transport Layer Security that helps authenticates the server and protects the transmitted data from tampering. ${ }^{61}$

\section{Data governance}

Data governance: To be able to reach effective data governance, this study will apply a Malaysia Data Protection Act 
2010 as its formal guidelines. This study will have a specific independent researcher in charge of data handling and managing. The independent researcher will be briefed on the policy guidelines to avoid any mishandling or mismanagement of the data.

Maintenance of anonymity: There will be an identifying number on KEPT-app downloaded during recruitment and follow-up from each participant to maintain anonymity in this research.

Data control: Using the Malaysian Data Protection Act 2010, only the researchers able to access the data, to ensure the data security, confidentiality and management strategy.

Data monitoring: The data will be given to the Universiti Putra Malaysia and ethical committee 6 monthly to update the research progress. All hard copies, for example, the consent forms with the digital recordings will be demolished and destroyed on the publication of this study' results (see online supplemental file 4). However, the researcher will keep only the research notes for 5 years following the publication of the study' results as this study' data will provide some background information for the future mHealth apps development.

\section{Dissemination}

The study protocol, instrument validation and KEPT-app results will be disseminated and published through conference presentations and peer-reviewed manuscripts. There is a potential interest to distribute the crucial findings via our university's website to introduce the importance of the KEPT-app.

\section{Publication policy}

Trial result: Each manuscript or abstract, must be submitted to all co-investigators for review of its appropriateness and scientific quality before submission. The manuscript must get permission from the Ministry of Health before publication.

\section{Dissemination policy: authorship}

The Primary Investigator will disseminate the suggested topics for presentation or publication to the coinvestigators. The writing committee will be formed to ensure that the writing process is systematic and discusses the results comprehensively. The coinvestigators will settle any disagreements regarding authorship after consultation with the primary investigator.

\section{Ethical issues and consideration(s)}

This study will be conducted in compliance with ethical principles outlined in the Declaration of Helsinki ${ }^{62}$ and Malaysian Good Clinical Practice Guideline. ${ }^{63}$ This research has its own Universal Trial Number by WHO: U1111-1228-5103. The study methods are conformed with the SPIRIT guidelines for reporting randomised trials. $^{2850}$

\section{Author affiliations}

${ }^{1}$ Department of Psychiatry, Faculty of Medicine and Health Sciences, Universiti Putra Malaysia, Serdang, Malaysia
${ }^{2}$ Primary Care Unit, Faculty of Medicine and Defence Health, Universiti Pertahanan Nasional Malaysia, Kuala Lumpur, Malaysia

${ }^{3}$ Department of Population Medicine, Universiti Tunku Abdul Rahman, Kajang, Malaysia

${ }^{4}$ Department of Family Medicine, Faculty of Medicine, Universiti Kebangsaan Malaysia, Kuala Lumpur, Malaysia

${ }^{5}$ Department of Community Health, Faculty of Medicine and Health Sciences, Universiti Putra Malaysia, Serdang, Malaysia

${ }^{6}$ Rehabilitation Department, KPJ Damansara Specialist Hospital, Damansara, Malaysia

${ }^{7}$ Computer Science Department, Faculty of Defence Science and Technology, Universiti Pertahanan Nasional Malaysia, Kuala Lumpur, Malaysia

${ }^{8}$ Selangor State Health Department, Hulu Langat District Health Office, Kajang, Malaysia

${ }^{9}$ Department of General Practice and Primary Health Care, University of Auckland, Auckland, New Zealand

Acknowledgements The researchers appreciate and acknowledge National Medical Research Registration committee for their management support.

Contributors SMS, AJ, CNF, SIFI, PA developed the original protocol. Later, FG-S, NAM, RAM, AFAF, ZS contributed to improving the study design. AJ wrote the protocol with considerable input from all authors at all stages. The contribution from all authors has been recorded and approved before the final manuscript.

Funding Universiti Putra Malaysia supported this work, 43400 UPM Serdang, Selangor, Malaysia grant number UPM/800-3/3/1/GPB/2018/9668500.

Disclaimer The opinions expressed are from the author(s) and not necessarily those of the Public Health Department or Ministry of Health. This funding source had no role in the design of this study and will not have any role during its execution, analyses, interpretation of the data, or decision to submit results.

\section{Competing interests None declared.}

Patient consent for publication Not required.

Provenance and peer review Not commissioned; externally peer reviewed.

Supplemental material This content has been supplied by the author(s). It has not been vetted by BMJ Publishing Group Limited (BMJ) and may not have been peer-reviewed. Any opinions or recommendations discussed are solely those of the author(s) and are not endorsed by BMJ. BMJ disclaims all liability and responsibility arising from any reliance placed on the content. Where the content includes any translated material, BMJ does not warrant the accuracy and reliability of the translations (including but not limited to local regulations, clinical guidelines, terminology, drug names and drug dosages), and is not responsible for any error and/or omissions arising from translation and adaptation or otherwise.

Open access This is an open access article distributed in accordance with the Creative Commons Attribution Non Commercial (CC BY-NC 4.0) license, which permits others to distribute, remix, adapt, build upon this work non-commercially, and license their derivative works on different terms, provided the original work is properly cited, appropriate credit is given, any changes made indicated, and the use is non-commercial. See: http://creativecommons.org/licenses/by-nc/4.0/.

\section{ORCID iDs}

Sherina Mohd Sidik http://orcid.org/0000-0001-6754-6145

Aida Jaffar http://orcid.org/0000-0003-2266-9509

Chai Nien Foo http://orcid.org/0000-0003-0362-6394

Felicity Goodyear-Smith http://orcid.org/0000-0002-6657-9401

\section{REFERENCES}

1 Abrams P, Cardozo L, Fall M, et al. The standardisation of terminology of lower urinary tract function: report from the standardisation Sub-committee of the International continence Society. Neurourol Urodyn 2002;21:167-78.

2 Irwin DE, Kopp ZS, Agatep B, et al. Worldwide prevalence estimates of lower urinary tract symptoms. BJU Int 2011;108:1132-9.

3 Ministry of Health Malaysia (MOH). Perinatal care manual 3 $R D$ edition. Malaysia: Division of Family Health Development., 2013: 1-251. http://fh.moh.gov.my/v3/index.php/component/ jdownloads/send/18-sektor-kesihatan-ibu/224-perinatal-caremanual-3rd-edition-2013? Itemid=0 
4 Hvidman L, Hvidman L, Foldspang A, et al. Correlates of urinary incontinence in pregnancy. Int Urogynecol J Pelvic Floor Dysfunct 2002;13:278-83.

5 Wesnes SL, Rortveit G, Bo K, et al. Urinary incontinence during pregnancy. Obstet Gynecol 2007;109:922-8.

6 Jaffar A, Mohd-Sidik S, Nien FC, et al. Urinary incontinence and its association with pelvic floor muscle exercise among pregnant women attending a primary care clinic in Selangor, Malaysia. PLoS One 2020;15:e0236140.

7 Coyne KS, Zhou Z, Thompson C, et al. The impact on health-related quality of life of stress, urge and mixed urinary incontinence. BJU Int 2003;92:731-5.

8 Abrams P, Andersson K-E, Apostolidis A, et al. 6Th International consultation on incontinence. recommendations of the International scientific Committee: evaluation and treatment of urinary incontinence, pelvic organ prolapse and faecal incontinence. Neurourol Urodyn 2018;37:2271-2.

9 Wesnes SL, Lose G. Preventing urinary incontinence during pregnancy and postpartum: a review. Int Urogynecol J 2013;24:889-99.

10 Sangsawang B, Sangsawang N. Stress urinary incontinence in pregnant women: a review of prevalence, pathophysiology, and treatment. Int Urogynecol J 2013;24:901-12.

11 Abdullah B, Ayub SH, Mohd Zahid AZ, et al. Urinary incontinence in primigravida: the neglected pregnancy predicament. Eur J Obstet Gynecol Reprod Biol 2016;198:110-5.

12 Burgio KL, Robinson JC, Engel BT. The role of biofeedback in Kegel exercise training for stress urinary incontinence. Am J Obstet Gynecol 1986;154:58-64.

13 Cavkaytar S, Kokanali MK, Topcu HO, et al. Effect of home-based Kegel exercises on quality of life in women with stress and mixed urinary incontinence. J Obstet Gynaecol 2015;35:407-10.

14 Schneider MS, King LR, Surwit RS. Kegel exercises and childhood incontinence: a new role for an old treatment. J Pediatr 1994;124:91-2

15 National Institute for Health and Care Excellence (UK). Urinary incontinence and pelvic organ prolapse in women: management, 2019. Available: www.nice.org.uk/guidance/ng123

16 Rosediani M, Juliawati M, Norwati D. Knowledge, attitude and practice towards pelvic floor muscle exercise among pregnant women attending antenatal clinic in Universiti Sains Malaysia Hospital, Malaysia. Int Med J 2012;19:37-8.

17 Frawley HC, McClurg D, Mahfooza A, et al. Health professionals' and patients' perspectives on pelvic floor muscle training adherence-2011 ICS State-of-the-Science seminar research paper IV of IV. Neurourol Urodyn 2015;34:632-9.

18 Bernard S, Boucher S, McLean L, et al. Mobile technologies for the conservative self-management of urinary incontinence: a systematic scoping review. Int Urogynecol J 2020;31:1163-74.

19 Leme Nagib AB, Riccetto C, Martinho NM, et al. Use of mobile apps for controlling of the urinary incontinence: a systematic review. Neurourol Urodyn 2020;39:1036-48.

20 Wang X, Xu X, Luo J, et al. Effect of app-based audio guidance pelvic floor muscle training on treatment of stress urinary incontinence in primiparas: a randomized controlled trial. Int J Nurs Stud 2020;104:103527.

21 National Institute for Health and Care Excellence. Urinary incontinence and pelvic organ prolapse in women: management. NICE guideline NG123, 2019. Available: https://www.nice.org. uk/guidance/ng123/chapter/Recommendations\#non-surgicalmanagement-of-urinary-incontinence

22 Woodley SJ, Lawrenson P, Boyle R, et al. Pelvic floor muscle training for preventing and treating urinary and faecal incontinence in antenatal and postnatal women. Cochrane Database Syst Rev 2020;5:CD007471.

23 Haff N, Choudhry NK. The promise and pitfalls of pragmatic clinical trials for improving health care quality. JAMA Netw Open 2018;1:e183376.

24 Health Informatics Centre M of HM. Health indicators 2018: indicators for monitoring and evaluation of strategy health for all. Ministry of health Malaysia (MOH), 2018. Available: https://www. aidsdatahub.org/resource/health-indicators-2018-indicatorsmonitoring-and-evaluation-strategy-health-all

25 Hayes RJ, Moulton LH. Cluster randomised trials. Boca Raton, Florida: Chapman and Hall/CRC, 2017.

$26 \mathrm{Kim}$ J, Shin W. How to do random allocation (randomization). Clin Orthop Surg 2014;6:103-9.

27 Campbell MK, Piaggio G, Elbourne DR, et al. Consort 2010 statement: extension to cluster randomised trials. BMJ 2012;345:e5661-21.
28 Chan A-W, Tetzlaff JM, Gøtzsche PC, et al. Spirit 2013 explanation and elaboration: guidance for protocols of clinical trials. $B M J$ 2013;346:e7586.

29 Zwarenstein M, Treweek S, Gagnier JJ, et al. Improving the reporting of pragmatic trials: an extension of the CONSORT statement. BMJ 2008;337:a2390.

30 Loudon K, Treweek S, Sullivan F, et al. The PRECIS-2 tool: designing trials that are fit for purpose. BMJ 2015;350:h2147.

31 Chalkidou K, Tunis S, Whicher D, et al. The role for pragmatic randomized controlled trials ( $\mathrm{pRCTs}$ ) in comparative effectiveness research. Clin Trials 2012;9:436-46.

32 Hopewell S, Clarke M, Moher D, et al. Consort for reporting randomised trials in Journal and conference abstracts. The Lancet 2008;371:281-3.

33 Kendall JM. Designing a research project: randomised controlled trials and their principles. Emerg Med J 2003;20:164-8.

34 National Institute for Health and Care Excellence (NICE). Antenatal care for uncomplicated pregnancies (CG62). London, 2008. Available: https://www.nice.org.uk/guidance/cg62

35 Koutoukidis DA, Lopes S, Atkins L, et al. Use of intervention mapping to adapt a health behavior change intervention for endometrial cancer survivors: the shape-up following cancer treatment program. BMC Public Health 2018;18:415.

36 Eldredge LKB, Markham CM, Ruiter RAC. Planning health promotion programs: an intervention mapping approach. Hoboken: John Wiley \& Sons, 2016.

37 Michie S, Atkins L, West R. The behaviour change wheel: a guide to designing interventions. 2014. Great Britain: Silverback Publishing, 2015.

38 Atkins L. Using the behaviour change wheel in infection prevention and control practice. J Infect Prev 2016;17:74-8.

39 Bandura A. Health promotion by social cognitive means. Health Educ Behav 2004;31:143-64.

40 Bo K, Berghmans B, Morkved S. Evidence-Based physical therapy for the pelvic floor-E-book: bridging science and clinical practice. United States of America: Elsevier Health Sciences, 2014.

41 de Santé HA. Good practice guidelines on health apps and smart devices (mobile health or mHealth), 2016. Available: https://www. has-sante.fr/upload/docs/application/pdf/2017-03/dir1/good practice_guidelines_on_health_apps_and_smart_devices_mobile_ health_or_mhealth.pdf

42 Calvert M, Blazeby J, Altman DG, et al. Reporting of patient-reported outcomes in randomized trials: the CONSORT pro extension. JAMA 2013;309:814-22.

43 Eldridge S, Kerry S. A practical guide to cluster randomised trials in health services research. Vol. 120. Hoboken: John Wiley \& Sons, 2012.

44 Donner A, Birkett N, Buck C. Randomization by cluster. sample size requirements and analysis. Am J Epidemiol 1981;114:906-14.

45 Eldridge SM, Kerry S. Sample size calculations [Internet]. A practical guide to cluster randomised trials in health services research. United Kingdom: Wiley Online Books, 2012: 137-71. https://doi.org/10. 1002/9781119966241.ch7

46 Campbell M, Grimshaw J, Steen N. Sample size calculations for cluster randomised trials. changing professional practice in Europe group (EU Biomed II concerted action). J Health Serv Res Policy 2000;5:12-16.

47 Ribeiro DC, Milosavljevic S, Abbott JH. Sample size estimation for cluster randomized controlled trials. Musculoskelet Sci Pract 2018;34:108-11.

48 Ramli AS, Selvarajah S, Daud MH, et al. Effectiveness of the EMPOWER-PAR intervention in improving clinical outcomes of type 2 diabetes mellitus in primary care: a pragmatic cluster randomised controlled trial. BMC Fam Pract 2016;17:157.

49 Eldridge SM, Ashby D, Kerry S. Sample size for cluster randomized trials: effect of coefficient of variation of cluster size and analysis method. Int J Epidemiol 2006;35:1292-300.

50 Chan A-W, Tetzlaff JM, Altman DG, et al. Spirit 2013 statement: defining standard protocol items for clinical trials. Ann Intern Med 2013;158:200-7.

51 Bell ML, Fiero M, Horton NJ, et al. Handling missing data in RCTs; a review of the top medical journals. BMC Med Res Methodol 2014;14:118.

52 Leyrat C, Morgan KE, Leurent B, et al. Cluster randomized trials with a small number of clusters: which analyses should be used? Int $J$ Epidemiol 2018;47:321-31.

53 Leyrat C, Caille A, Eldridge S, et al. Intervention effect estimates in cluster randomized versus individually randomized trials: a metaepidemiological study. Int J Epidemiol 2019;48:609-19.

54 Morel JG, Bokossa MC, Neerchal NK. Small sample correction for the variance of Gee estimators. Biometrical J 2003;45:395-409. 
55 Asklund I, Nyström E, Sjöström M, et al. Mobile APP for treatment of stress urinary incontinence: a randomized controlled trial. Neurourol Urodyn 2017;36:1369-76.

56 Heissel A, Pietrek A, Schwefel M, et al. STEP.De study-a multicentre cluster-randomised effectiveness trial of exercise therapy for patients with depressive symptoms in healthcare services: study protocol. BMJ Open 2020;10:e036287.

57 Spieth PM, Kubasch AS, Penzlin Al, et al. Randomized controlled trials - a matter of design. Neuropsychiatr Dis Treat 2016;12:1341-9.

58 Moher D, Hopewell S, Schulz KF, et al. Consort 2010 explanation and elaboration: updated guidelines for reporting parallel group randomised trials. Int J Surg 2012;10:28-55.

59 Laws of Malaysia. Personal data potection act 2010, 2016. Available: http://www.agc.gov.my/agcportal/uploads/files/Publications/LOM/ EN/Act 709146 2016.pdf

60 Frunza M, Scripcariu L. Improved RSA encryption algorithm for increased security of wireless networks. In: 2007 International Symposium on signals, circuits and systems. IEEE 2007;2:1-4.

61 Boyd C, Mathuria A, Stebila D. Transport layer security protocol $B T$ - protocols for authentication and key establishment. Berlin, Heidelberg: Springer Berlin Heidelberg, 2020: 241-88.

62 General Assembly of the World Medical Association. World Medical association declaration of Helsinki: ethical principles for medical research involving human subjects. J Am Coll Dent 2014;81:14.

63 National Pharmaceutical Regulatory Agency (NPRA), Ministry of Health, Malaysia. Malaysian guideline for good clinical practice 4th edition. Malaysia: National Committee for Clinical Research (NCCR), 2018.

64 Nyström E, Sjöström M, Stenlund H, et al. ICIQ symptom and quality of life instruments measure clinically relevant improvements in women with stress urinary incontinence. Neurourol Urodyn 2015;34:747-51.
65 Muhammad J, Muhamad R, Husain NRN, et al. Pelvic floor muscle exercise education and factors associated with implementation among antenatal women in hospital Universiti Sains Malaysia Korean J Fam Med 2019;40:45-52.

66 Lemeshow S, Hosmer DW, Klar J, et al. Adequacy of sample size in health studies. Chichester: Wiley, 1990.

67 Avery K, Donovan J, Peters TJ, et al. ICIQ: a brief and robust measure for evaluating the symptoms and impact of urinary incontinence. Neurourol Urodyn 2004;23:322-30.

68 Newman-Beinart NA, Norton S, Dowling D, et al. The development and initial psychometric evaluation of a measure assessing adherence to prescribed exercise: the exercise adherence rating scale (ears). Physiotherapy 2017;103:180-5.

69 Kroenke K, Spitzer RL, Williams JB. The PHQ-9: validity of a brief depression severity measure. J Gen Intern Med 2001;16:606-13.

70 Sherina MS, Arroll B, Goodyear-Smith F. Criterion validity of the PHQ-9 (Malay version) in a primary care clinic in Malaysia. Med $J$ Malaysia 2012;67:309-15.

71 Sidebottom AC, Harrison PA, Godecker A, et al. Validation of the Patient Health Questionnaire (PHQ)-9 for prenatal depression screening. Arch Womens Ment Health 2012;15:367-74.

72 Spitzer RL, Kroenke K, Williams JBW, et al. A brief measure for assessing generalized anxiety disorder: the GAD-7. Arch Intern Med 2006;166:1092-7.

73 Kroenke K, Spitzer RL, Williams JBW, et al. Anxiety disorders in primary care: prevalence, impairment, comorbidity, and detection. Ann Intern Med 2007;146:317-25.

74 Zhong Q-Y, Gelaye B, Zaslavsky AM, et al. Diagnostic validity of the generalized anxiety disorder - 7 (GAD-7) among pregnant women. PLoS One 2015;10:e0125096.

75 Sidik SM, Arroll B, Goodyear-Smith F. Validation of the GAD-7 (Malay version) among women attending a primary care clinic in Malaysia. $J$ Prim Health Care 2012;4:5-11, A1. 